\title{
LOOP VARIABLES FOR COMPACT TWO-DIMENSIONAL QUANTUM ELECTRODYNAMICS
}

\author{
Rodolfo Gambini ${ }^{\dagger}$, Hugo A. Morales-Técotl ${ }^{*}$, , \\ Luis F. Urrutia ${ }^{\ddagger}$ and J. David Vergara ${ }^{\ddagger}$. \\ †Departamento de Física, Facultad de Ciencias, \\ Universidad de La República, \\ Tristán Narvaja 1674, Montevideo. \\ *Departamento de Física, Universidad Autónoma Metropolitana-Iztapalapa, \\ Apartado Postal 55-534, 09340, México D.F. \\ §International Centre for Theoretical Physics, \\ P.O. Box 586, 34100 Trieste. \\ ¥Instituto de Ciencias Nucleares, Universidad Nacional Autónoma de México, \\ Apartado Postal 70-543, 04510, México D.F.
}

\begin{abstract}
Variables parametrized by closed and open curves are defined to reformulate compact U(1) Quantum Electrodynamics in the circle with a massless fermion field. It is found that the gauge invariant nature of these variables accommodates into a regularization scheme for the Hamiltonian and current operators that is specially well suited for the study of the compact case. The zero mode energy spectrum, the value of the axial anomaly and the anomalous commutators this model presents are hence determined in a manifestly gauge invariant manner. Contrary to the non compact case, the zero mode spectrum is not equally spaced and consequently the theory does not lead to the spectrum of a free scalar boson. All the states are invariant under large gauge transformations. In particular, that is the case for the vacuum, and consequently the $\theta$-dependence does not appear.
\end{abstract}

03.70, 11.15, 11.40.H

Typeset using REVTEX 


\section{INTRODUCTION}

Back in 1962 Schwinger considered the possibility that a vector gauge field can imply a nonzero mass gauge particle. He showed this is indeed the case for an exactly solvable model, namely two-dimensional Quantum Electrodynamics $\left(\mathrm{QED}_{2}\right)$ with a massless fermion field [囬]. This simple, although non-trivial, field theory has become since an arena to probe different aspects of quantum field theory [2,3]. For instance, the axial anomaly, the charge screening and the bosonization presented by the model, together with alternative methods of solution, have been studied by different authors 4 -9. Of particular interest are the results from $\mathrm{QED}_{2}$ that might shed light on the non-perturbative features not only of $\mathrm{QED}_{4}$ but also of $\mathrm{QCD}_{4}$ and quantum gravity in its gauge-theory-like formulation [10 12].

Being $\mathrm{QED}_{4}$ the gauge theory par excellence it was considered worth adopting loop variables techniques in its description [13,11] because in this way gauge invariance could be explicitly implemented. Lattice $\mathrm{QED}_{4}$ has been successfully developed along these lines and computational calculations have been improved with respect to simulations [14]. Also, a fourdimensional (continuum) gravitational analogue constructed out of gravity plus fermions has been studied in this framework [15]. In every case the presence of fermions is automatically accounted for by including open curves, besides loops, to parametrize operators and state vectors. The fermions necessarily stand at the end points of the open curves. Moreover, the dynamics gets geometrically coded into the breaking, rejoining and rerouting of such open curves and loops through their intersection points. This is deeply significant for nonperturbative quantum gravity where the role of diffeomorphism invariance is central and such a description naturally fits in [15]. For the $\mathrm{QED}_{4}$ case this geometrical picture of dynamics led to useful criteria to approximate the strong coupling regime [14].

Wilson loops are computed in terms of the holonomy elements associated to the parallel transport along closed curves. In the Maxwell case the holonomies are elements of the U(1) group. They are not only invariant under small gauge transformations generated by the Gauss law constraint, but also under large gauge transformations. That is the reason why they naturally describe compact electrodynamics, which is also characterized by the property that the range of $A_{1}(x)$, in the Weyl gauge for the two-dimensional case, is the circle instead of the real line as it is in the standard noncompact case. This fact is well known and has far reaching consequences in higher dimensions. Polyakov [16 has argued that it is necessary to decide, based on physical grounds, what version of QED is realized in nature. In particular, the fact that in the non abelian case the non compact version cannot be formulated on a discrete lattice leads him to consider that, if QED arises as a subgroup of some nonabelian gauge theory, we are necessarily dealing with the compact version. Earlier discussions of compact QED in the lattice can be found in Refs. [17], for example. The Schwinger model has been recently studied in the hamiltonian [18] and lagrangean $19 \|$ lattice loop representation. In these papers, it is shown that the chiral symmetry is broken and the $\theta$-dependence of the vacuum is not present.

In what concerns the case of higher dimensions, it has been shown that monopoles arising in the compact abelian sector of $2+1$ QCD play a fundamental role in the confinement process. In Polyakov's analysis loops are crucial to understand this process. In particular, he has recently shown that the loop world sheets acquires string like degrees of freedom due to the presence of a diluted gas of monopoles [20].

In this work, loop variables are introduced for the compact Schwinger's model along the lines of [14,15]. Starting with the canonical analysis of $\mathrm{QED}_{2}$, which yields the Gauss law first-class constraint, loop variables are defined such that they have zero Poisson brackets with it. They form a closed algebra and turn out to be enough to describe the dynamics. The Hamiltonian is reexpressed as a limit of some of these loop variables when the curves shrink down to a point. The quantum theory is defined such that the Poisson algebra becomes a commutator algebra and the loop representation is built by choosing one of these loop operators to create a state with an extra loop (open curve) out of an arbitrary state and then using the operator algebra. Thus, it is possible to work entirely in the loop 
representation. In order to recover the standard (local) physical information, like the energy spectrum for example, one has to take the corresponding limit of the loops (open curves) shrinking to a point. Before determining the properties of the energy spectrum of the full theory, we found it convenient to study the semiclassical situation where there is a quantum fermion field interacting with a classical electromagnetic field. Some technicalities become more transparent if use is made of a bi-local Fourier transform of the operators parametrized by open curves. Details are given in the appendix. This procedure yields a built-in gaugeinvariant point-split regularization for the fermion vacuum energy. In this semiclassical context, the vacuum to vacuum expectation value for the divergence of the axial current produces the known value for the axial anomaly in a straightforward manner. Also, the vacuum expectation value of the so called anomalous commutators are directly derived from the loop variables algebra. Remarkably enough, the corresponding Poisson brackets algebra already contains the relevant information. Using the separability of the full Schroedinger equation for the system, the zero mode sector of the spectrum is considered next. The nonequally spaced results for the zero mode energy found in this case seem to indicate that, in the general compact case, the spectrum does not correspond to a free massive boson. By considering the limit in which the length of the $S^{1}$ spatial slice goes to zero one recovers the typical harmonic oscillator spectrum for the zero mass mode together with its free bosonic behavior, which is characteristic of the non-compact Schwinger Model.

As we have previously emphasized, loop variables naturally describe a compact version of the electromagnetic interaction. In order to recover the noncompact theory it is necessary to introduce additional angular variables, which are conjugated to the integer numbers characterizing the large gauge transformations. A detailed discussion of this important issue can be found in Ref. [21.

The organization of the paper is as follows. In section II we translate the local classical dynamics of $\mathrm{QED}_{2}$ into loop variables. The corresponding algebra is displayed there. The quantum counterpart is then exhibited in section III, where state functionals are loop/curve parametrized; hence defining the loop representation. In section IV, the analysis is carried out taking the fermion field as a quantum entity evolving in the external electromagnetic field. Hereby the fermion vacuum is found. In section V, the well known chiral anomaly coming from the non-conservation of the axial current is computed. Section VI contains the calculation of the anomalous commutators. The zero mode sector of the theory is finally analyzed in section VII based on the external electromagnetic field approach of the previous section. Finally, section VIII contains some general remarks on the loop approach for $\mathrm{QED}_{2}$ together with possible future developments. The appendix presents the explicit relation between loop operators and their useful bi-local Fourier transforms, making more transparent the analysis here presented.

\section{CLASSICAL FRAMEWORK}

Our starting point is the real Lagrangian density

$$
\mathcal{L}=-\frac{1}{4} F_{\mu \nu} F^{\mu \nu}+\frac{\hbar}{2} \bar{\psi} \gamma^{\mu}\left(i \partial_{\mu}-e A_{\mu}\right) \psi-\frac{\hbar}{2}\left[\left(i \partial_{\mu}+e A_{\mu}\right) \bar{\psi}\right] \gamma^{\mu} \psi
$$

where $F_{\mu \nu}=\partial_{\mu} A_{\nu}-\partial_{\nu} A_{\mu}$ and $\bar{\psi}=\psi^{\dagger} \gamma^{0}$ is a Grassmann valued fermionic field. Since space here is $S^{1}$, we will require periodic(antiperiodic) boundary conditions for the fields

$$
A_{\mu}(x+L)=A_{\mu}(x), \quad \psi(x+L)=-\psi(x),
$$

where $L=2 \pi r$ is the length of the circle. The gamma matrices are: $\gamma^{0}=\sigma_{1}, \gamma^{1}=$ $-i \sigma_{2}, \gamma^{5}=\gamma^{0} \gamma^{1}=\sigma_{3}$, where $\sigma_{i}$ are the standard Pauli matrices. We use the signature $(+,-)$, i.e. $\eta_{00}=-\eta_{11}=1$. 
The Lagrangian density (11) is invariant under the following gauge transformations

$$
\psi \rightarrow e^{i e \alpha(x, t)} \psi, \quad A_{\mu} \rightarrow A_{\mu}-\partial_{\mu} \alpha(x, t) .
$$

There are two families of gauge transformations: (i) those continuously connected to the identity, called small gauge transformations, characterized by the function $\alpha=b(t) e^{i 2 \pi n x / L}$ which is periodic in $x$ and preserves the boundary conditions (2). The second family corresponds to the so called large gauge transformations, which is determined by the non-periodic functions $\alpha=\frac{2 \pi n}{e L} x, n= \pm 1, \pm 2, \ldots$. The boundary conditions (21) are also preserved in this case.

After the standard canonical analysis the Hamiltonian density becomes

$$
\mathcal{H}=\frac{1}{2} E^{2}-\frac{i \hbar}{2} \psi^{\dagger} \sigma_{3}\left(\partial_{1}+i e A\right) \psi+\frac{i \hbar}{2}\left[\left(\partial_{1}-i e A\right) \psi^{*}\right] \sigma_{3} \psi-A_{0} \mathcal{G} .
$$

Here $E=F_{01}, A=A_{1}$ and

$$
\mathcal{G}=\partial_{1} E-e \hbar \psi^{*} \psi
$$

is the Gauss law constraint. The boundary term associated with the integration of $\partial_{x}\left(E A_{0}\right)$ yields no contribution to the Hamiltonian because of the boundary conditions on the vector potential $A_{\mu}$. In what follows $\psi=\left(\psi_{1}, \psi_{2}\right)^{\top}, \top$ denoting transposition. The charge density is given by $\rho(x)=e\left(\psi_{1}^{*} \psi_{1}+\psi_{2}^{*} \psi_{2}\right)$. We are working in units such that $c=1, \hbar \neq 1$ and we take mass $[\mathrm{g}]$ and length $[\mathrm{cm}]$ as the basic ones. In this way the corresponding dimensions are: $\hbar=[g \mathrm{~cm}], E=\left[\sqrt{\frac{g}{c m}}\right], A=[\sqrt{g \mathrm{~cm}}], \quad e A=\left[\frac{1}{\mathrm{~cm}}\right], \psi_{1}^{*} \psi_{1}=\left[\frac{1}{\mathrm{~cm}}\right], \psi_{2}^{*} \psi_{2}=\left[\frac{1}{\mathrm{~cm}}\right]$ and $\hbar e^{2} L^{2}$ is a dimensionless quantity. Both the combinations $\frac{\hbar}{L}$ and $e \hbar^{\frac{3}{2}}$ have the dimensions of mass.

The resulting Poisson brackets algebra at equal times is

$$
\begin{aligned}
\{A(x), E(y)\} & =\delta(x, y) \\
\left\{\psi_{\alpha}(x), \psi_{\beta}^{*}(y)\right\} & =-\frac{i}{\hbar} \delta_{\alpha \beta} \delta(x, y) \quad \alpha=1,2 .
\end{aligned}
$$

\section{A. Loop variables}

In order to take into account the Gauss law ab initio, one can adopt the following gauge invariant non local variables [14:

$$
\begin{aligned}
T^{0}(\gamma) & =\exp \left\{i e \oint_{\gamma} d x A(x)\right\}, \\
\Pi_{0}\left(\eta_{x}{ }^{y}\right) & =\psi_{1}^{*}(x) U\left(\eta_{x}{ }^{y}\right) \psi_{2}(y), \\
\Pi_{1}\left(\eta_{x}{ }^{y}\right) & =\psi_{1}^{*}(x) U\left(\eta_{x}{ }^{y}\right) \psi_{1}(y), \\
\Pi_{2}\left(\eta_{x}{ }^{y}\right. & =\psi_{2}^{*}(x) U\left(\eta_{x}{ }^{y}\right) \psi_{2}(y), \\
\Pi_{3}\left(\eta_{x}{ }^{y}\right) & =\psi_{2}^{*}(x) U\left(\eta_{x}{ }^{y}\right) \psi_{1}(y),
\end{aligned}
$$

with $U\left(\eta_{x}^{y}\right)=\exp \left\{i e \int_{\eta_{x} y} d z A(z)\right\}$ and, of course, $E(x)$ which is gauge invariant by construction. The open paths $\eta_{x}^{y}$ are always arcs of circumference starting at the point $x$ and ending at the point $y$. No independent loop variable is obtained by considering a gauge invariant non local variable containing $E(x)$ as an insertion. 
It is important to recall, once again, that in this approach all the information about the theory is encoded in terms of loop variables, which are gauge invariant under small and large gauge transformations. That means, in particular, that the electromagnetic information is encoded in the elements of the $\mathrm{U}(1)$ group $U\left(\eta_{x}^{y}\right)$ and consequently the loop representation naturally describes compact electrodynamics. In other words, since the basic electromagneric variable is $\exp i e \int_{0}^{L} d x A(x)$, it is enough to restrict $\int_{0}^{L} d x A(x)$ to the interval $\left[0, \frac{2 \pi}{e}\right]$.

The induced non zero Poisson brackets among the loop variables are

$$
\begin{aligned}
& \left\{T^{0}(\gamma), E(x)\right\}=i e \oint_{\gamma} d z \delta(x, z) T^{0}(\gamma), \\
& \left\{\Pi_{i}\left(\eta_{x}^{y}\right), E(z)\right\}=i e \int_{\eta_{x} y} d u \delta(z, u) \Pi_{i}\left(\eta_{x}^{y}\right), \quad i=0,1,2,3, \\
& \left\{\Pi_{0}\left(\alpha_{x}^{y}\right), \Pi_{1}\left(\eta_{u}^{v}\right)\right\}=\frac{i}{\hbar} \delta(x, v) \Pi_{0}\left((\eta \circ \alpha)_{u}^{y}\right), \\
& \left\{\Pi_{0}\left(\alpha_{x}^{y}\right), \Pi_{2}\left(\eta_{u}{ }^{v}\right)\right\}=-\frac{i}{\hbar} \delta(u, y) \Pi_{0}\left((\alpha \circ \eta)_{x}^{v}\right), \\
& \left\{\Pi_{0}\left(\alpha_{x}^{y}\right), \Pi_{3}\left(\eta_{u}^{v}\right)\right\}=\frac{i}{\hbar} \delta(x, v) \Pi_{2}\left((\eta \circ \alpha)_{u}^{y}\right), \\
& -\frac{i}{\hbar} \delta(y, u) \Pi_{1}\left((\alpha \circ \eta)_{x}^{v}\right) \text {, } \\
& \left\{\Pi_{i}\left(\alpha_{x}^{y}\right), \Pi_{i}\left(\eta_{u}{ }^{v}\right)\right\}=-\frac{i}{\hbar} \delta(y, u) \Pi_{i}\left((\alpha \circ \eta)_{x}^{v}\right)+ \\
& \frac{i}{\hbar} \delta(x, v) \Pi_{i}\left((\eta \circ \alpha) u^{y}\right), i=1,2, \\
& \left\{\Pi_{1}\left(\alpha_{x}^{y}\right), \Pi_{3}\left(\eta_{u}{ }^{v}\right)\right\}=\frac{i}{\hbar} \delta(x, v) \Pi_{3}\left((\eta \circ \alpha)_{u}^{y}\right), \\
& \left\{\Pi_{2}\left(\alpha_{x}^{y}\right), \Pi_{3}\left(\eta_{u}{ }^{v}\right)\right\}=-\frac{i}{\hbar} \delta(y, u) \Pi_{3}\left((\alpha \circ \eta)_{x}{ }^{v}\right) .
\end{aligned}
$$

Since it will be crucial later on, we stress here that the line integral $\int_{\eta_{x} y} d u \delta(u, z)$ is more conveniently expressed as [9]

$$
\theta(x, y ; z) \equiv \int_{\eta_{x} y} d u \delta(u, z)=\frac{1}{L}\left(y-x+\sum_{k \neq 0} \frac{1}{i k}\left(e^{i k(y-z)}-e^{i k(x-z)}\right)\right)
$$

where $k=\frac{2 \pi n}{L}, n= \pm 1, \pm 2, \ldots$. We observe that $\lim _{x \rightarrow y} \theta(x, y ; z)=0$. We can verify also that

$$
\frac{\partial \theta(x, y ; z)}{\partial z}=\delta(x, z)-\delta(y, z)
$$

The representation of the delta function is given by

$$
\delta(x, y)=\frac{1}{L} \sum_{n=-\infty}^{+\infty} e^{\frac{2 \pi i n}{L}(x-y)}
$$

and the following properties can be directly obtained

$$
\delta(x, y)=\delta(y, x), \quad \delta(x, 0)=\delta(x, L) .
$$


To proceed further we rewrite $\mathcal{H}, \mathcal{G}, \rho$ in terms of loop variables

$$
\begin{aligned}
\mathcal{H}(x) & =\frac{1}{2} E^{2}(x)+\frac{i \hbar}{2} \lim _{y \rightarrow x}\left(\partial_{x}-\partial_{y}\right)\left(\Pi_{2}\left(\alpha_{y}{ }^{x}\right)-\Pi_{1}\left(\alpha_{y}{ }^{x}\right)\right) \\
\mathcal{G}(x) & =\partial_{x} E(x)-e \hbar \lim _{y \rightarrow x}\left(\Pi_{1}\left(\alpha_{y}{ }^{x}\right)+\Pi_{2}\left(\alpha_{y}{ }^{x}\right)\right) \\
\rho(x) & =\lim _{y \rightarrow x} e\left(\Pi_{1}\left(\alpha_{y}{ }^{x}\right)+\Pi_{2}\left(\alpha_{y}{ }^{x}\right)\right)
\end{aligned}
$$

Although the quantities $\mathcal{H}, \rho$ are gauge invariant by construction in terms of the local fields, one can directly verify that their Poisson brackets with the Gauss constraint are identically zero using the above algebra, i.e. Eqs. (12-19). Because it is illuminating we next show that the charge $Q=\int_{0}^{L} d w \rho(w)$ is a constant of motion

$$
\begin{aligned}
\{Q, H\}= & e \int_{0}^{L} d w \int_{0}^{L} d y \lim _{\substack{z \rightarrow w \\
x \rightarrow y}}\left[E(y) i e \theta(z, w ; y)\left(\Pi_{1}\left(\eta_{z}{ }^{w}\right)+\Pi_{2}\left(\eta_{z}{ }^{w}\right)\right)\right. \\
& +i \partial_{y}\left(-i \delta(w, x) \Pi_{2}\left((\eta \circ \alpha)_{z}{ }^{y}\right)+i \delta(z, y) \Pi_{2}\left((\alpha \circ \eta)_{x}{ }^{w}\right)\right) \\
& \left.-i \partial_{y}\left(-i \delta(w, x) \Pi_{1}\left((\eta \circ \alpha)_{z}{ }^{y}\right)+i \delta(z, y) \Pi_{1}\left((\alpha \circ \eta)_{x}{ }^{w}\right)\right)\right]=0 .
\end{aligned}
$$

The Hamiltonian is $H=\int_{0}^{L} d x \mathcal{H}$. Note that the first line of (27) is zero because of $\lim _{z \rightarrow w} \theta(z, w ; y)=0$, provided $\lim _{z \rightarrow w} \Pi_{+}(z, w)$ is finite. That the second line is also zero can be readily seen as follows: after taking the limits $x \rightarrow y, z \rightarrow w$ in the term $\partial_{y} \delta(z, y) \Pi_{2}((\alpha \circ$ $\left.\eta)_{x}{ }^{w}\right)$ and integrating by parts the first term $\delta(w, y) \partial_{y} \Pi_{2}\left(\alpha_{w}{ }^{y}\right)$ gets cancelled. The remaining boundary term is zero after performing the second integral. The third line is zero for analogous reasons.

\section{QUANTUM FRAMEWORK}

Our general procedure of quantization will be to promote the observables $A, B$ to operators $\hat{A}, \hat{B}$ and let their Poisson brackets $\{A, B\}=C$ go over (anti)commutators $[\hat{A}, \hat{B}]_{ \pm}=i \hbar \hat{C}$ according to the standard prescription.

\section{A. Local field representation}

We define this representation by choosing the configuration variables as $\hat{A}(x), \hat{\psi}_{1}^{*}(x)$, $\hat{\psi}_{2}(x)$ acting multiplicatively on a wave function $\Psi\left(A(x), \psi_{1}^{*}(x), \psi_{2}(x)\right)$. The remaining variables will be represented by:

$$
\hat{E}(x)=-i \hbar \frac{\delta}{\delta A(x)}, \quad \hat{\psi}_{1}=\frac{\delta}{\delta \psi_{1}^{*}(x)}, \quad \hat{\psi}_{2}^{*}=\frac{\delta}{\delta \psi_{2}(x)} .
$$

In this representation, the state of nothing $|0\rangle$ which does not contain any field excitation, is given by a constant functional.

\section{B. Loop representation}

This representation will be constructed as a quantum realization of the loop variables Poisson algebra given by Eqs.(12-19). To this end, it is convenient to first characterize the 
state of nothing $|0\rangle$, which is not to be confused with the physical vacuum of the theory. The former is characterized by $\left\langle A, \psi_{1}^{*}, \psi_{2} \mid 0\right\rangle=$ const. in the previous representation. This leads us to define the state of nothing as

$$
\hat{E}(x)|0\rangle=0, \quad \hat{\Pi}_{i}(x, y)|0\rangle=0, \quad i=1,2,3 .
$$

Note that these conditions enforce the gauge invariance of this state. The above definitions imply that, in the connection representation, the functional derivative operator must always go to the right. In this way, we choose the following representation for the gauge invariant operators $\hat{\Pi}_{i}\left(\eta_{x}^{y}\right)$

$$
\begin{aligned}
& \hat{\Pi}_{0}\left(\eta_{x}{ }^{y}\right)=\psi_{1}^{*}(x) U\left(\eta_{x}{ }^{y}\right) \psi_{2}(y), \\
& \hat{\Pi}_{1}\left(\eta_{x}^{y}\right)=\psi_{1}^{*}(x) U\left(\eta_{x}^{y}\right) \frac{\delta}{\delta \psi_{1}^{*}(y)}, \\
& \hat{\Pi}_{2}\left(\eta_{x}{ }^{y}\right)=-\psi_{2}(y) U\left(\eta_{x}^{y}\right) \frac{\delta}{\delta \psi_{2}(x)}, \\
& \hat{\Pi}_{3}\left(\eta_{x}^{y}\right)=\frac{\delta}{\delta \psi_{2}(x)} U\left(\eta_{x}^{y}\right) \frac{\delta}{\delta \psi_{1}^{*}(y)},
\end{aligned}
$$

where the minus sign in $\hat{\Pi}_{2}$ has been introduced to recover the classical limit of Eq.(10). Note that $\hat{\Pi}_{3}^{\dagger}(x, y)=\hat{\Pi}_{0}(y, x)$ in the standard scalar product of Grassmann variables.. The resulting non-zero commutators are

$$
\begin{aligned}
{\left[\hat{\Pi}_{0}(u, v), \hat{\Pi}_{1}(x, y)\right]=} & -\delta(u, y) \hat{\Pi}_{0}(x, v), \\
{\left[\hat{\Pi}_{0}(u, v), \hat{\Pi}_{2}(x, y)\right]=} & \delta(x, v) \hat{\Pi}_{0}(u, y), \\
{\left[\hat{\Pi}_{0}(u, v), \hat{\Pi}_{3}(x, y)\right]=} & \delta(x, v) \hat{\Pi}_{1}(u, y)-\delta(u, y) \hat{\Pi}_{2}(x, v) \\
& -\delta(u, y) \delta(x, v), \\
{\left[\hat{\Pi}_{i}(u, v), \hat{\Pi}_{i}(x, y)\right]=} & \delta(x, v) \hat{\Pi}_{i}(u, y)-\delta(u, y) \hat{\Pi}_{i}(x, v), \quad i=1,2 \\
{\left[\hat{\Pi}_{+}(u, v), \hat{\Pi}_{+}(x, y)\right]=} & \delta(x, v) \hat{\Pi}_{+}(u, y)-\delta(u, y) \hat{\Pi}_{+}(x, v), \\
{\left[\hat{\Pi}_{-}(u, v), \hat{\Pi}_{-}(x, y)\right]=} & \delta(x, v) \hat{\Pi}_{+}(u, y)-\delta(u, y) \hat{\Pi}_{+}(x, v), \\
{\left[\hat{\Pi}_{+}(u, v), \hat{\Pi}_{-}(x, y)\right]=} & \delta(x, v) \hat{\Pi}_{-}(u, y)-\delta(u, y) \hat{\Pi}_{-}(x, v), \\
{\left[\hat{\Pi}_{3}(u, v), \hat{\Pi}_{1}(x, y)\right]=} & \delta(x, v) \hat{\Pi}_{3}(u, y), \\
{\left[\hat{\Pi}_{3}(u, v), \hat{\Pi}_{2}(x, y)\right]=} & -\delta(u, y) \hat{\Pi}_{3}(x, v), \\
{\left[\hat{\Pi}_{i}(u, v), \hat{E}(x)\right]=} & -e \hbar \theta(u, v ; x) \hat{\Pi}_{i}(u, v), \quad i=0,1,2,3 .
\end{aligned}
$$

where the c-number contribution in Eq.(36) arises from the ordering of the operators. In the above equations we have introduced the notation

$$
\hat{\Pi}_{ \pm}(x, y):=\hat{\Pi}_{1}(x, y) \pm \hat{\Pi}_{2}(x, y)
$$

and from now on we denote $\hat{\Pi}_{i}\left(\eta_{x}^{y}\right)$ simply by $\hat{\Pi}_{i}(x, y)$.

An heuristic application of the loop transform shows that the operators $\hat{T}^{0}(\gamma)$ together with $\hat{\Pi}_{0}\left(\eta_{x}^{y}\right)$ acting on the state of nothing $\mid 0>$, create states with closed and open curves respectively [13,15]. This can be formally stated as

$$
\begin{aligned}
\hat{T}^{0}\left(\gamma_{1}\right) \ldots \hat{T}^{0}\left(\gamma_{q}\right)|0\rangle & =\left|\gamma_{1}, \ldots, \gamma_{q}\right\rangle \\
\hat{\Pi}_{0}\left(x_{1}, y_{1}\right) \ldots \hat{\Pi}_{0}\left(x_{m}, y_{m}\right)|0\rangle & =\left|x_{1}, y_{1}, \ldots, x_{m}, y_{m}\right\rangle .
\end{aligned}
$$


Hence, the commutators of the operators with $\hat{\Pi}_{0}$ will provide the action of such operators upon generic states. For simplicity we will consider states like $|\gamma\rangle$ and $|\ldots, x, y, \ldots\rangle$ separately. The basic idea is to apply the corresponding commutator to the state of nothing. Suppose we want to calculate $\hat{E}(x)|\gamma\rangle$. To this end let us consider

$$
\begin{aligned}
{\left[\hat{E}(x), \hat{T}^{0}(\gamma)\right]|0\rangle } & =e \hbar \oint_{\gamma} d u \delta(x, u) \hat{T}^{0}(\gamma)|0\rangle \\
\hat{E}(x)|\gamma\rangle & =e \hbar \oint_{\gamma} d u \delta(x, u)|\gamma\rangle=e \hbar n|\gamma\rangle
\end{aligned}
$$

where $n$ is the winding number of the closed curve $\gamma$. In a completely analogous way we obtain

$$
\hat{E}(z)\left|x_{1}, y_{1}, \ldots, x_{m}, y_{m}\right\rangle=e \hbar\left(\sum_{k=1}^{m} \theta\left(x_{k}, y_{k} ; z\right)\right)\left|x_{1}, y_{1}, \ldots, x_{m}, y_{m}\right\rangle .
$$

Let us observe that in spite of Eq.(45), which would demand $q$ labels $n_{q}$, we need only one label counting the total winding number $n=n_{1}+\ldots+n_{q}$. This is because $\hat{E}(x)\left|n_{1}, n_{2}\right\rangle=$ $e \hbar\left(n_{1}+n_{2}\right)\left|n_{1}, n_{2}\right\rangle \approx \hat{E}(x)\left|n_{1}+n_{2}\right\rangle$, which allow us to identify the states $\left|n_{1}, n_{2}\right\rangle$ and $\left|n_{1}+n_{2}\right\rangle$ up to a phase. Thus, we identify $\left|\gamma_{1}, \ldots, \gamma_{q}\right\rangle=|n\rangle$.

The remaining operators $\hat{\Pi}_{i}, i=1,2,3$, leave invariant the state associated to the closed curve $\gamma$, while some examples of their action upon states defined by open curves are

$$
\begin{aligned}
\hat{\Pi}_{1}(w, u)|x, y\rangle & =\delta(x, u)|w, y\rangle \\
\hat{\Pi}_{2}(w, u)|x, y\rangle & =-\delta(y, w)|x, u\rangle \\
\hat{\Pi}_{3}(u, v)|x, y\rangle= & \delta(x, v) \delta(u, y)|0\rangle \\
\hat{\Pi}_{3}(u, v)|x, y, w, z\rangle= & -\delta(x, v) \delta(z, u)|w, y\rangle-\delta(y, u) \delta(w, v)|x, z\rangle \\
& +\delta(u, y) \delta(x, v)|w, z\rangle+\delta(u, z) \delta(v, w)|x, y\rangle
\end{aligned}
$$

In this way, we consider our Hilbert space to be spanned by the set of all vectors

$$
\left|n ; x_{1} y_{1}, \ldots, x_{a}, y_{a}\right\rangle \equiv|n\rangle \otimes\left|x_{1} y_{1}, \ldots, x_{a}, y_{a}\right\rangle,-\infty<n<+\infty, a=0,1,2 \ldots, \infty .
$$

These vectors satisfy the following orthogonality and closure properties

$$
\begin{aligned}
& \left\langle n ; x_{1}, y_{1}, \ldots, x_{a}, y_{a} \mid m ; u_{1} v_{1}, \ldots, u_{b}, v_{b}\right\rangle=\delta_{m, n} \delta_{a, b} \\
& \sum_{q_{i}, p_{j}} \epsilon_{q_{1} q_{2} \ldots q_{a}} \epsilon_{p_{1} p_{2} \ldots p_{a}} \delta\left(x_{1}-u_{q_{1}}\right) \delta\left(y_{1}-v_{p_{1}}\right) \ldots \delta\left(x_{a}-u_{q_{a}}\right) \delta\left(y_{a}-v_{p_{a}}\right), \\
& \sum_{n=-\infty}^{+\infty} \sum_{a=0}^{+\infty} \int d x_{1} d y_{1} \ldots d x_{a} d y_{a} \frac{1}{a !^{2}}\left|n ; x_{1}, y_{1}, \ldots, x_{a}, y_{a}\right\rangle\left\langle n ; x_{1}, y_{1}, \ldots, x_{a}, y_{a}\right|=1
\end{aligned}
$$

which are a direct consequence of the basic algebra (34)-(42). They also satisfy the following exchange properties

$$
\begin{aligned}
\left|n ; \ldots, x_{i}, y_{i}, \ldots, x_{j}, y_{j}, \ldots x_{a} y_{a}\right\rangle & =\left|n ; \ldots, x_{j}, y_{j}, \ldots, x_{i}, y_{i}, \ldots, x_{a}, y_{a}\right\rangle \\
\left|n ; \ldots, x_{i}, y_{i}, \ldots, x_{j}, y_{j}, \ldots x_{a} y_{a}\right\rangle & =-\left|n ; \ldots, x_{j}, y_{i}, \ldots, x_{i}, y_{j}, \ldots x_{a} y_{a}\right\rangle \\
\left|n ; \ldots, x_{i}, y_{i}, \ldots, x_{j}, y_{j}, \ldots x_{a} y_{a}\right\rangle & =-\left|n ; \ldots, x_{i}, y_{j}, \ldots, x_{j}, y_{i}, \ldots x_{a} y_{a}\right\rangle
\end{aligned}
$$


Equation (53) is a direct consequence of the definition in Eq. (46) and the fact that $\left[\hat{\Pi}_{0}\left(x_{i}, y_{i}\right), \hat{\Pi}_{0}\left(x_{j}, y_{j}\right)\right]=0$, while Eqs. (54) and (55) arise from the anticommuting property of the fermion operators together with the abelian composition rule

$$
U(u, v) U(x, y)=U(u, y) U(x, v) .
$$

In the loop representation, a general wave function $|\Psi\rangle$ can be written as

$$
|\Psi\rangle=\sum_{a=0}^{\infty} \sum_{n=-\infty}^{+\infty} \int d x_{1} d y_{1} \ldots d x_{a} d y_{a} \frac{1}{a !} \Psi^{a}\left(n ; x_{1}, y_{1}, \ldots, x_{a}, y_{a}\right)\left|n ; x_{1} y_{1}, \ldots, x_{a}, y_{a}\right\rangle
$$

where

$$
\left\langle n ; x_{1}, y_{1}, \ldots, x_{a}, y_{a} \mid \Psi\right\rangle=\Psi^{a}\left(n ; x_{1}, y_{1}, \ldots, x_{a}, y_{a}\right)
$$

are the corresponding components. They inherit the exchange properties (53), (54) and (55) of the basis vectors and also satisfy the following boundary conditions

$$
\begin{aligned}
\Psi^{a}\left(n ; \ldots, x_{k}, y_{k}+m L, \ldots\right) & =e^{i m \pi} \Psi^{a}\left(n+m ; \ldots, x_{k}, y_{k}, \ldots\right), \\
\Psi^{a}\left(n ; \ldots, x_{k}+s L, y_{k}, \ldots\right) & =e^{-i s \pi} \Psi^{a}\left(n-s ; \ldots, x_{k}, y_{k}, \ldots\right),
\end{aligned}
$$

which arise from the property $\Pi_{0}(x, y+m L)=e^{i m \pi} \psi_{1}^{*}(x) U(x, y) T^{0}(m) \psi_{2}(y)$, in virtue of the boundary conditions (2). As a consequence of the symmetry properties (59) and (60) we will assume from here on that $0 \leq x_{k}, y_{k}<L$.

The scalar product is given by

$$
\begin{aligned}
\langle\Phi \mid \Psi\rangle=\sum_{a=0}^{\infty} \sum_{n=-\infty}^{+\infty} \sum_{i_{a}} \int d x_{1} d y_{1} \ldots d x_{a} d y_{a}\left(\Phi^{a}\left(n ; x_{1}, y_{1}, \ldots, x_{a}, y_{a}\right)\right)^{*} \\
\times \Psi^{a}\left(n ; x_{1}, y_{i_{1}}, \ldots, x_{a}, y_{i_{a}}\right)
\end{aligned}
$$

where the functions $\Phi^{a}\left(n ; x_{1}, y_{1}, \ldots, x_{a}, y_{a}\right)$ are the components of the wave function $|\Phi\rangle$.

In order to find the associated Schroedinger equation we need to compute the action of the operators appearing in the Hamiltonian density (24) upon the basis vectors. We obtain

$$
\begin{aligned}
\hat{E}^{2}(x)\left|n ; x_{1}, y_{1}, \ldots, x_{a}, y_{a}\right\rangle & =e^{2} \hbar^{2}\left[n+\sum_{k=1}^{a} \theta\left(x_{k}, y_{k}, x\right)\right]^{2}\left|n ; x_{1}, y_{1}, \ldots, x_{a}, y_{a}\right\rangle, \\
\hat{\Pi}_{1}(x, y)\left|n ; x_{1}, y_{1}, \ldots, x_{a}, y_{a}\right\rangle & =\hbar \sum_{k=1}^{a} \delta\left(x_{k}, y\right)\left|n ; \ldots, x_{k-1}, y_{k-1}, x, y_{k}, x_{k+1}, y_{k+1}, \ldots, x_{a}, y_{a}\right\rangle, \\
\hat{\Pi}_{2}(x, y)\left|n ; x_{1}, y_{1}, \ldots, x_{a}, y_{a}\right\rangle & =-\hbar \sum_{k=1}^{a} \delta\left(y_{k}, x\right)\left|n ; \ldots, x_{k-1}, y_{k-1}, x_{k}, y, x_{k+1}, y_{k+1}, \ldots, x_{a}, y_{a}\right\rangle .
\end{aligned}
$$

Using the above actions, we have explicitly verified that the basis vectors (50) are annihilated by the Gauss law constraint (25).

The Hamiltonian $\hat{H}$ is block-diagonal in the subspace of fixed number of pairs and fixed $n$. Thus we look for solutions of the Schroedinger equation, $\hat{H}|\Psi\rangle=E|\Psi\rangle$, which are of the form 


$$
|\Psi\rangle_{a, n}=\int d x_{1} d y_{1} \ldots d x_{a} d y_{a} \Psi^{a}\left(n ; x_{1}, y_{1}, \ldots, x_{a}, y_{a}\right)\left|n ; x_{1} y_{1}, \ldots, x_{a}, y_{a}\right\rangle
$$

The action upon the components of the wave function is

$$
\begin{aligned}
\left\langle n ; x_{1}, y_{1}, \ldots, x_{a}, y_{a}|\hat{H}| \Psi\right\rangle_{a, n}=\frac{e^{2} \hbar^{2}}{2}\left(\int_{0}^{L} d x\left[n+\sum_{k=1}^{a} \theta\left(x_{k}, y_{k}, x\right)\right]^{2}\right) \Psi^{a}\left(n ; x_{1}, y_{1}, \ldots, x_{a}, y_{a}\right) \\
+i \frac{\hbar}{2} \int_{0}^{L} d x \sum_{k=1}^{a} \partial_{x} \delta\left(x_{k}, x\right) \Psi^{a}\left(n ; \ldots, x_{k-1}, y_{k-1}, x, y_{k}, x_{k+1}, y_{k+1}, \ldots, x_{a}, y_{a}\right) \\
+i \frac{\hbar}{2} \int_{0}^{L} d x \sum_{k=1}^{a} \delta\left(y_{k}, x\right) \partial_{x} \Psi^{a}\left(n ; \ldots, x_{k-1}, y_{k-1}, x_{k}, x, x_{k+1}, y_{k+1}, \ldots, x_{a}, y_{a}\right) \\
\quad-i \frac{\hbar}{2} \int_{0}^{L} d x \sum_{k=1}^{a} \partial_{x} \delta\left(y_{k}, x\right) \Psi^{a}\left(n ; \ldots, x_{k-1}, y_{k-1}, x_{k}, x, x_{k+1}, y_{k+1}, \ldots, x_{a}, y_{a}\right) \\
\quad-i \frac{\hbar}{2} \int_{0}^{L} d x \sum_{k=1}^{a} \delta\left(x_{k}, x\right) \partial_{x} \Psi^{a}\left(n ; \ldots, x_{k-1}, y_{k-1}, x, y_{k}, x_{k+1}, y_{k+1}, \ldots, x_{a}, y_{a}\right) .
\end{aligned}
$$

Integrating by parts the term containing the derivative of the delta function in (66) we obtain the final result

$$
\begin{aligned}
\left\langle n ; x_{1}, y_{1}, \ldots, x_{a}, y_{a}|\hat{H}| \Psi\right\rangle_{a, n}= & \frac{e^{2} \hbar^{2}}{2}\left(\int_{0}^{L} d x\left[n+\sum_{k=1}^{a} \theta\left(x_{k}, y_{k}, x\right)\right]^{2}\right) \Psi^{a}\left(n ; x_{1}, y_{1}, \ldots, x_{a}, y_{a}\right) \\
& -i \hbar \sum_{k=1}^{a}\left(\frac{\partial}{\partial x_{k}}-\frac{\partial}{\partial y_{k}}\right) \Psi^{a}\left(n ; x_{1}, y_{1}, \ldots, x_{k}, y_{k}, \ldots, x_{a}, y_{a}\right) .
\end{aligned}
$$

\section{EXTERNAL FIELD ANALYSIS}

As a first step in the quantization of the full system we consider the quantization of the fermionic fields in a background electromagnetic field. According to Ref. [8], the fermionic field operators are given by

$$
\psi_{1}(x, t)=\sum_{n} a_{n} \phi_{n}(x) e^{-\frac{i}{\hbar} \epsilon_{n} t}, \quad \psi_{2}(x, t)=\sum_{n} b_{n}^{\dagger} \phi_{n}(x) e^{\frac{i}{\hbar} \epsilon_{n} t}
$$

where we have slightly changed the notation in the second equation (68). The operators $a_{n}, b_{n}$ are standard fermionic annihilation operators satisfying the non-zero anticommutators: $\left\{a_{n}, a_{m}^{\dagger}\right\}=\delta_{m n}=\left\{b_{m}, b_{n}^{\dagger}\right\}$. The basic wave functions $\phi_{n}$, together with the eigenvalues of the energy are given by

$$
\phi_{n}(x)=\frac{1}{\sqrt{L}} e^{\frac{i}{\hbar} \epsilon_{n} x-i e \int_{0}^{x} A(z) d z}, \quad \frac{1}{\hbar} \epsilon_{n}=\frac{2 \pi}{L}\left(n+\frac{1}{2}+\frac{e L}{2 \pi} c\right) \equiv \frac{2 \pi n}{L}+\theta,
$$

where

$$
c=\frac{1}{L} \oint A(z) d z, \quad \theta=\frac{\pi}{L}+e c
$$


The energy eigenvalues are invariant under small gauge transformations in such a way that the corresponding eigenfunctions transform covariantly according to (3). The same structure is kept in the case of large gauge transformations. To see this, it is enough to recall that in the compact $\mathrm{U}(1)$ case under consideration $c$ lies in the interval $\left[0, \frac{2 \pi}{e L}\right]$. This means that $c$ is invariant under large gauge transformations.

In the scalar product

$$
(\phi, \xi)=\int_{0}^{L} d z[\phi(z)]^{*} \xi(z),
$$

the functions defined in (69) are orthonormal, i.e. $\left(\phi_{m}, \phi_{n}\right)=\delta_{m n}$.

It is convenient to introduce a bi-local Fourier transform of the loop space operators $\hat{\Pi}(x, y)$ in the following way

$$
\Pi^{m n}=\int \frac{d x d y}{L} e^{\frac{i}{\hbar} \epsilon_{m} x} e^{-\frac{i}{\hbar} \epsilon_{n} y} \hat{\Pi}(x, y),
$$

together with its inverse

$$
\hat{\Pi}(x, y)=\frac{1}{L} \sum_{m, n} e^{-\frac{i}{\hbar} \epsilon_{m} x} e^{\frac{i}{\hbar} \epsilon_{n} y} \Pi^{m n} .
$$

Also, we define the Fourier transform of the electric field operator $E_{b}$, as

$$
E(x)=\sum_{b=-\infty}^{\infty} E_{b} e^{-\frac{2 \pi i b}{L} x}, \quad E_{b}^{\dagger}=E_{-b}, \quad b=0, \pm 1, \pm 2, \ldots
$$

which leads to the following inverse transformations

$$
E_{b}=\frac{1}{L} \int_{0}^{L} d x E(x) e^{\frac{2 \pi i b}{L} x} .
$$

The commutator algebra (3.7)-(3.13) can be directly rewritten in terms of the Fouriertransformed operators $\Pi^{m n}$ and $E_{b}$. The result is

$$
\begin{aligned}
& {\left[\Pi_{0}^{m n}, \Pi_{1}^{k l}\right]=-\delta^{m l} \Pi_{0}^{k n},} \\
& {\left[\Pi_{0}^{m n}, \Pi_{2}^{k l}\right]=\delta^{k n} \Pi_{0}^{m l},} \\
& {\left[\Pi_{i}^{m n}, \Pi_{i}^{k l}\right]=\delta^{k n} \Pi_{i}^{m l}-\delta^{m l} \Pi_{i}^{k n}, \quad i=1,2,} \\
& {\left[\Pi_{+}^{m n}, \Pi_{-}^{k l}\right]=\delta^{k n} \Pi_{-}^{m l}-\delta^{m l} \Pi_{-}^{k n},} \\
& {\left[\Pi_{3}^{m n}, \Pi_{1}^{k l}\right]=\delta^{k n} \Pi_{3}^{m l},} \\
& {\left[\Pi_{3}^{m n}, \Pi_{2}^{k l}\right]=-\delta^{m l} \Pi_{3}^{k n},} \\
& {\left[\Pi_{0}^{m n}, \Pi_{3}^{k l}\right]=\delta^{k n} \Pi_{1}^{m l}-\delta^{m l} \Pi_{2}^{k n}-\delta^{k n} \delta^{m l},}
\end{aligned}
$$

where $\Pi_{3}^{m n \dagger}=\Pi_{0}^{n m}$.

The commutators involving the electric modes are

$$
\begin{aligned}
{\left[E_{a}, E_{b}\right] } & =0, \quad\left[c, E_{b}\right]=\frac{i \hbar}{L} \delta_{b 0}, \\
{\left[T^{0}(n), E_{b}\right] } & =-e n \hbar T^{0}(n) \delta_{b 0}, \quad\left[\Pi_{i}^{k l}, E_{0}\right]=0, \quad i=0,1,2,3, \\
{\left[\Pi_{i}^{k l}, E_{b}\right] } & =\frac{i e \hbar}{2 \pi b}\left(\Pi_{i}^{k l-b}-\Pi_{i}^{k+b l}\right), b \neq 0 .
\end{aligned}
$$




\section{A. Vacuum state in a background electromagnetic field}

The Hamiltonian density that describes the external field approximation is given by the the second term in the RHS of (24). When written in the momentum space, the corresponding Hamiltonian is

$$
H_{D}=\sum_{m} \epsilon_{m} \Pi_{-}^{m m}
$$

The calculation of the commutators

$$
\left[\Pi_{0}^{k l}, H_{D}\right]=-\left(\epsilon_{k}+\epsilon_{l}\right) \Pi_{0}^{k l}, \quad\left[\Pi_{3}^{k l}, H_{D}\right]=+\left(\epsilon_{k}+\epsilon_{l}\right) \Pi_{3}^{k l},
$$

implies that $\Pi_{0}^{k l}$ is an energy raising operator, i.e. $\Pi_{0}^{k l}|E\rangle \sim\left|E+\epsilon_{k}+\epsilon_{l}\right\rangle$, for any Hamiltonian eigenstate $|E\rangle$. For analogous reasons, $\Pi_{3}^{k l}$ is the corresponding lowering operator. The charge operator $Q$ commutes with $\Pi_{0}^{k l}$, showing that $\Pi_{0}^{k l}$ creates a zero charge pair of particles having the corresponding energies $\epsilon_{k}$ and $\epsilon_{l}$. Thus we can identify each superindex of $\Pi_{0}^{k l}$ with a definite and opposite charge label. In this way we have that $\Pi_{0}^{k l} \Pi_{0}^{k r}|E\rangle \sim\left|E+2 \epsilon_{k}+\epsilon_{l}+\epsilon_{r}\right\rangle$. Since in one spatial dimension the momentum is proportional to the energy, we conclude that the state $\left|E+2 \epsilon_{k}+\epsilon_{l}+\epsilon_{r}\right\rangle$ contains two fermions having the same quantum numbers and therefore must be zero according to the Pauli principle. Since the eigenstates of the Hamiltonian provide a basis for the Hilbert space, we must have the operator identity

$$
\Pi_{0}^{k l} \Pi_{0}^{k r}=0
$$

and analogously, when the repeated indices are those in the right.

The vacuum state $|0\rangle_{D}$ corresponds to a filled Dirac sea with zero charge which can be defined as

$$
|0\rangle_{D}=\prod_{k=-\infty}^{N-1} \Pi_{0}^{k k}|0\rangle, \quad{ }_{D}\langle 0|=\langle 0| \prod_{k=-\infty}^{N-1} \Pi_{3}^{k k}
$$

This means that all energy levels below $\epsilon_{N}$ are completely filled. Provided that $-\left(N+\frac{1}{2}\right) \leq$ $\frac{e L}{2 \pi} c \leq-\left(N-\frac{1}{2}\right)$, we have that $\epsilon_{N} \geq 0$ and the above construction includes an infinite set of negative-energy states $\epsilon_{N-1} \leq 0$.

From now on we will use the convention that all indices ranging from $-\infty$ to $N-1$ will be denoted by capital letters from the beginning of the alphabet $(A, B, C, \ldots)$, while those going from $N$ to $+\infty$ will be denoted by lower case greek letters from the beginning of the alphabet $(\alpha, \beta, \gamma, \ldots)$.

The commutation relations (76), (77) imply that

$$
\begin{aligned}
& \Pi_{1}^{k k}\left(\Pi_{0}^{m m}\right)|0\rangle=\delta^{m k}\left(\Pi_{0}^{m m}\right)|0\rangle \\
& \Pi_{2}^{k k}\left(\Pi_{0}^{m m}\right)|0\rangle=-\delta^{m k}\left(\Pi_{0}^{m m}\right)|0\rangle,
\end{aligned}
$$

which allow us to prove that the vacuum satisfies

$$
\begin{gathered}
\Pi_{1}^{A A}|0\rangle_{D}=|0\rangle_{D}, \quad \Pi_{2}^{A A}|0\rangle_{D}=-|0\rangle_{D} \\
\Pi_{1}^{\alpha \alpha}|0\rangle_{D}=0=\Pi_{2}^{\alpha \alpha}|0\rangle_{D} .
\end{gathered}
$$

Next we discuss some additional properties of the vacuum. To begin with let us calculate $\Pi_{+}^{A B}|0\rangle_{D}$. We have already shown that $\Pi_{+}^{A A}|0\rangle_{D}=0, \forall A$. Now, for the case $A \neq B$, we have 


$$
\begin{aligned}
\Pi_{+}^{A B}|0\rangle_{D} & =\Pi_{+}^{A B} \prod_{C=-\infty}^{N-1} \Pi_{0}^{C C}|0\rangle=\left[\Pi_{+}^{A B}, \prod_{C=-\infty}^{N-1} \Pi_{0}^{C C}\right]|0\rangle \\
& =\sum_{D=-\infty}^{N-1} \prod_{s=-\infty}^{D-1} \Pi_{0}^{C C}\left[\Pi_{+}^{A B}, \Pi_{0}^{D D}\right] \prod_{G=D+1}^{N-1} \Pi_{0}^{G G}|0\rangle .=0
\end{aligned}
$$

The last equality holds by virtue of the commutation relation

$$
\left[\Pi_{+}^{A B}, \Pi_{0}^{C D}\right]=\delta^{B C} \Pi_{0}^{A D}-\delta^{A D} \Pi_{0}^{B C},
$$

together with the fact that for $A \neq B$ one will always find products of the type $\Pi_{0}^{A A} \Pi_{0}^{A B}$, $\Pi_{0}^{A A} \Pi_{0}^{B A}$, which are identically zero, according to the property (86). Thus, we have proved that $\Pi_{+}^{A B}|0\rangle_{D}=0$. Analogously one can show that $\Pi_{+}^{\alpha \beta}|0\rangle_{D}=0$. Nevertheless, both states $\Pi_{+}^{A \beta}|0\rangle_{D}$ together with $\Pi_{+}^{\alpha B}|0\rangle_{D}$ are different from zero and are in fact orthogonal to $|0\rangle_{D}$. Thus, we have

$$
{ }_{D}\left\langle 0\left|\Pi_{+}(x, y)\right| 0\right\rangle_{D}=0 .
$$

Next, we repeat the calculation for $\Pi_{-}(x, y)$. Again, we start from the "momentumspace" formulation. The properties (90) , (91) imply

$$
\Pi_{-}^{A A}|0\rangle_{D}=2|0\rangle_{D}, \quad \Pi_{-}^{\alpha \alpha}|0\rangle_{D}=0
$$

for the diagonal terms. When $A \neq B$, following analogous steps to the previous case, we find

$$
\Pi_{-}^{A B}|0\rangle_{D}=\sum_{C=-\infty}^{N-1} \prod_{D=-\infty}^{C-1} \Pi_{0}^{D D}\left[\Pi_{-}^{A B}, \Pi_{0}^{C C}\right] \prod_{G=C+1}^{N-1} \Pi_{0}^{G G}|0\rangle .
$$

The corresponding commutator here is

$$
\left[\Pi_{-}^{A B}, \Pi_{0}^{C D}\right]=\delta^{B C} \Pi_{0}^{A D}+\delta^{A D} \Pi_{0}^{B C} .
$$

Once more, we obtain $\Pi_{-}^{A B}|0\rangle_{D}=0, A \neq B$ because of the presence of products of $\Pi_{0}^{\prime} s$ having a repeated index. In analogous way one obtains the remaining actions leading to

$$
\Pi_{-}^{A B}|0\rangle_{D}=2 \delta^{A B}|0\rangle_{D}, \quad \Pi_{-}^{\alpha \beta}|0\rangle_{D}=0, \quad \Pi_{-}^{A \beta}|0\rangle_{D} \neq 0, \quad \Pi_{-}^{\alpha B}|0\rangle_{D} \neq 0 .
$$

Again, the non-zero vectors resulting from the last two actions in the above equation are orthogonal to the Dirac vacuum. In this way, going back to the coordinate representation we obtain

$$
{ }_{D}\left\langle 0\left|\Pi_{-}(x, y)\right| 0\right\rangle_{D}=2 e^{-i \theta(x-y)} \frac{1}{L} \sum_{A=-\infty}^{N-1} e^{-\frac{2 \pi i A}{L}(x-y)}{ }_{D}\langle 0 \mid 0\rangle_{D}=\frac{2}{L} e^{-i \theta(x-y)} F(x, y)_{D}\langle 0 \mid 0\rangle_{D} .
$$

In the above equation we have introduced the function $F$ defined as

$$
F(x, y)=\sum_{A=-\infty}^{N-1} e^{-\frac{2 \pi i A}{L}(x-y)}=\frac{e^{-\frac{2 \pi i(N-1)}{L}(x-y)}}{\left(1-e^{\frac{2 \pi i}{L}(x-y)}\right)}
$$

where the summation can be calculated because it is a geometric series. 


\section{B. The vacuum energy}

Let us recall that the Hamiltonian is given by

$$
H_{D}=\frac{i \hbar}{2} \int_{0}^{L} d x \lim _{y \rightarrow x}\left[\left(\partial_{y}-\partial_{x}\right) \Pi_{-}(y, x)\right]
$$

According to the relations (98) and (100) the action of $\Pi_{-}(y, x)$ on the Dirac vacuum can be written as

$$
\Pi_{-}(x, y)|0\rangle_{D}=\frac{2}{L} e^{-i \theta(x-y)} F(x, y)|0\rangle_{D}+|x, y ;-\rangle
$$

where the state $|x, y ;-\rangle$ does not contributes in the limit of Eq.(101). The corresponding vacuum energy in the external field is given by

$$
E_{D}(\epsilon)=\frac{\left\langle 0\left|\hat{H}_{D}\right| 0\right\rangle_{D}}{\langle 0 \mid 0\rangle_{D}}
$$

The function $E_{D}(\epsilon)$ will have an expansion in powers of $\epsilon$ of the form $E_{D}(\epsilon)=\frac{a}{\epsilon^{2}}+b \epsilon^{0}+O(\epsilon)$. We will take $b$ as the regularized expression for the vacuum energy $E_{D}$. The resulting term is

$$
E_{D}(\epsilon)=2 i \hbar \partial_{\epsilon}\left[e^{-i \theta \epsilon} \frac{e^{-\frac{2 \pi i(N-1)}{L} \epsilon}}{1-e^{\frac{2 \pi i}{L} \epsilon}}\right]
$$

The finite part of the above equation, when $\epsilon \rightarrow 0$, is

$$
E_{D}=\hbar\left[\frac{2 \pi N^{2}}{L}-\frac{2 \pi N}{L}+2 N \theta-\theta+\frac{\pi}{3 L}+\frac{\theta^{2} L}{2 \pi}\right]
$$

which coincides with the result of Ref. 88. In the sequel we choose $N=0$, in such a way that $-\pi \leq e c L \leq+\pi$, which reinforces the fact that $e c$ is a compact degree of freedom.

\section{THE AXIAL ANOMALY}

The vector and axial currents are defined as:

$$
\begin{aligned}
J_{V}^{\mu}(x) & :=\lim _{y \rightarrow x} e \bar{\psi}(y) \gamma^{\mu} U(y, x) \psi(x), \\
J_{A}{ }^{\mu}(x) & :=\lim _{y \rightarrow x} e \bar{\psi}(y) \gamma^{\mu} \gamma_{5} U(y, x) \psi(x) .
\end{aligned}
$$

Thus, in terms of loop variables, their components become

$$
\begin{array}{ll}
J_{V}^{0}=\lim _{y \rightarrow x} e \Pi_{+}(y, x), & J_{V}^{1}=\lim _{y \rightarrow x} e \Pi_{-}(y, x), \\
J_{A}^{0}=\lim _{y \rightarrow x} e \Pi_{-}(y, x), & J_{A}^{1}=\lim _{y \rightarrow x} e \Pi_{+}(y, x) .
\end{array}
$$

Next we calculate the relevant commutators in order to determine $\left[\hat{Q}_{A, V}, \hat{H}\right]$, where 


$$
\hat{Q}_{V}:=\int_{0}^{L} d x \lim _{y \rightarrow x} e \hat{\Pi}_{+}(y, x), \quad \hat{Q}_{A}:=\int_{0}^{L} d x \lim _{y \rightarrow x} e \hat{\Pi}_{-}(y, x)
$$

and $\hat{H}$ is the full Hamiltonian. To do so we start by looking at the following commutators

$$
\left[\hat{\Pi}_{ \pm}(y, x), \hat{H}\right]=-\frac{e \hbar}{2} \int_{x}^{y} d w\left(\hat{E}(w) \hat{\Pi}_{ \pm}(y, x)+\hat{\Pi}_{ \pm}(y, x) \hat{E}(w)\right)-i \hbar\left(\partial_{x}+\partial_{y}\right) \hat{\Pi}_{\mp}(y, x),
$$

In obtaining the above commutators, we have used the property

$$
\delta(x, L) \hat{\Pi}_{ \pm}(y, L)-\delta(x, 0) \hat{\Pi}_{ \pm}(y, 0)=0=\delta(y, L) \hat{\Pi}_{ \pm}(L, x)-\delta(y, 0) \hat{\Pi}_{ \pm}(0, x),
$$

according to Eq. (22). Let us consider now $\int_{0}^{L} d x \lim _{y \rightarrow x}\left[\hat{\Pi}_{1}(y, x), \hat{H}\right]$. One can readily see that the integral

$$
\int_{0}^{L} d x \lim _{y \rightarrow x}\left(\partial_{x}+\partial_{y}\right) \hat{\Pi}_{ \pm}(y, x)=\lim _{\epsilon \rightarrow 0} \int_{0}^{L} d x \partial_{x} \hat{\Pi}_{ \pm}(x+\epsilon, x)=\hat{\Pi}_{ \pm}(L, L)-\hat{\Pi}_{ \pm}(0,0)=0 .
$$

The only term left is

$$
\int_{0}^{L} d x \lim _{y \rightarrow x}\left[\hat{\Pi}_{ \pm}(y, x), \hat{H}\right]=-\frac{e \hbar}{2} \lim _{\epsilon \rightarrow 0} \int_{0}^{L} d x \int_{x}^{x+\epsilon} d w\left[\hat{E}(w) \hat{\Pi}_{ \pm}(x+\epsilon, x)+\hat{\Pi}_{ \pm}(x+\epsilon, x) \hat{E}(w)\right] .
$$

Thus, the above limit would yield zero provided $\hat{\Pi}_{ \pm}(x, x)$ is finite. Nevertheless we expect $\hat{\Pi}_{ \pm}(x, x)$ to be divergent so that the limit must be carefully calculated.

In order to obtain the divergence of the vector current we start from

$$
\partial_{0} \hat{J}_{V}^{0}=\frac{i}{\hbar}\left[\hat{H}, \hat{J}_{V}^{0}\right] .
$$

The calculation of the commutator leads to

$$
\partial_{0} \hat{J}_{V}^{0}=\lim _{y \rightarrow x} \frac{i e^{2}}{2} \int_{x}^{y} d w\left(\hat{E}(w) \hat{\Pi}_{+}(y, x)+\hat{\Pi}_{+}(y, x) \hat{E}(w)\right)-\lim _{y \rightarrow x}\left(\partial_{x}+\partial_{y}\right) e \hat{\Pi}_{-}(y, x) .
$$

After taking the limit, the second term in the r.h.s. in (116) can be identified as $-\partial_{1} \hat{J}_{V}{ }^{1}$. This can be shown by going to the momentum representation: on one hand we have that

$$
\partial_{1} \hat{J}_{V}{ }^{1}=\partial_{x} \lim _{y \rightarrow x} e \hat{\Pi}_{-}(y, x)=\frac{e}{\hbar L} \sum_{m, n} i\left(\epsilon_{m}-\epsilon_{n}\right) e^{\frac{i}{\hbar}\left(\epsilon_{m}-\epsilon_{n}\right) x} \Pi_{-}^{m n} .
$$

On the other hand, the limit appearing in Eq.(116) is calculated as

$$
\lim _{y \rightarrow x}\left(\partial_{x}+\partial_{y}\right) e \hat{\Pi}_{-}(y, x)=\lim _{y \rightarrow x} \frac{e}{\hbar L} \sum_{m, n} i\left(\epsilon_{m}-\epsilon_{n}\right) e^{\frac{i}{\hbar} \epsilon_{m} x} e^{-\frac{i}{\hbar} \epsilon_{n} y} \Pi_{-}^{m n},
$$

which reduces exactly to Eq.(117) after taking the limit $y \rightarrow x$.

Hence the final result in the calculation of Eq.(116) is

$$
\partial_{\mu} \hat{J}_{V}^{\mu}=\lim _{y \rightarrow x} \frac{i e^{2}}{2} \int_{x}^{y} d w\left(\hat{E}(w) \hat{\Pi}_{+}(y, x)+\hat{\Pi}_{+}(y, x) \hat{E}(w)\right) .
$$


In analogous way we obtain

$$
\partial_{\mu} \hat{J}_{A}^{\mu}=\lim _{y \rightarrow x} \frac{i e^{2}}{2} \int_{x}^{y} d w\left(\hat{E}(w) \hat{\Pi}_{-}(y, x)+\hat{\Pi}_{-}(y, x) \hat{E}(w)\right) .
$$

Let us remark that Eqs. (119), (120) are full operator relations which describe the exact non-perturbative behavior of the divergences of the corresponding currents.

In order to recover the standard form of the axial anomaly, we assume that only the fermions are quantized, and calculate the vacuum expectation values of the above divergences of the currents regarding $E(x)$ as an external field, together with the vacuum given by Eqs.(90, 91). Using Eq.(94) and Eq.(99) we obtain

$$
\begin{gathered}
{ }_{D}\left\langle 0\left|\partial_{\mu} \hat{J}_{V}{ }^{\mu}\right| 0\right\rangle_{D}=0, \\
\left\langle 0\left|\partial_{\mu} \hat{J}_{A}{ }^{\mu}\right| 0\right\rangle_{D}=\lim _{y \rightarrow x} i e^{2} E(x)(y-x)_{D}\left\langle 0\left|\Pi_{-}(y, x)\right| 0\right\rangle_{D}, \\
\frac{\left\langle 0\left|\partial_{\mu} \hat{J}_{A}{ }^{\mu}\right| 0\right\rangle_{D}}{\langle 0 \mid 0\rangle_{D}}=\frac{2}{L} i e^{2} E(x) \lim _{y \rightarrow x}\left(\frac{(y-x) e^{\frac{2 \pi i}{L}(y-x)}}{\left(1-e^{\frac{2 \pi i}{L}(y-x)}\right)}\right)=-\frac{e^{2}}{\pi} E(x) .
\end{gathered}
$$

\section{ANOMALOUS COMMUTATORS}

It is a general property of quantum field theory that, if a current is conserved, the equal time commutator of its spatial and temporal components cannot vanish [22]. However, by a naive use of the canonical commutation relations one finds that this commutator is equal to zero. To obtain the correct result it is necessary to introduce a regularization. The nonlocality of our formalism provides a natural regularization and the algebra (34-12) produces directly the anomalous commutators. For example, we have

$$
\begin{aligned}
{\left[J_{V}^{0}(u), J_{V}^{1}(x)\right] } & =\left[\lim _{v \rightarrow u} e \hat{\Pi}_{+}(v, u), \lim _{y \rightarrow x} e \hat{\Pi}_{-}(y, x)\right] \\
& =e^{2} \lim _{v \rightarrow u} \lim _{y \rightarrow x}\left(\delta(y-u) \hat{\Pi}_{-}(v, x)-\delta(v-x) \hat{\Pi}_{-}(y, u)\right) .
\end{aligned}
$$

Taking the limits $v=u+\epsilon$ and $y=x+\epsilon$, with $\epsilon \rightarrow 0$, the vacuum expectation value of the commutator has the form

$$
{ }_{D}\left\langle 0\left|\left[J_{V}^{0}(u), J_{V}^{1}(x)\right]\right| 0\right\rangle_{D}=\frac{i e^{2}}{\pi} \delta^{\prime}(x-u) .
$$

Also, from the relations (43), it follows that the commutator of the currents and the electric field is different of zero, which agree with the Gauss law (25). In this case we have

$$
\begin{aligned}
& {\left[J_{V}^{0}(x), E(z)\right]=\left[\lim _{y \rightarrow x} e \hat{\Pi}_{+}(x, y), \hat{E}(z)\right]=-e^{2} \lim _{y \rightarrow x} \theta(x, y ; z) \hat{\Pi}_{+}(x, y),} \\
& {\left[J_{V}^{1}(x), E(z)\right]=\left[\lim _{y \rightarrow x} e \hat{\Pi}_{-}(x, y), \hat{E}(z)\right]=-e^{2} \lim _{y \rightarrow x} \theta(x, y ; z) \hat{\Pi}_{-}(x, y),}
\end{aligned}
$$

From the above expressions we obtain the vacuum expectation value of these commutators

$$
\begin{gathered}
{ }_{D}\left\langle 0\left|\left[J_{V}^{0}(x), E(z)\right]\right| 0\right\rangle_{D}=0, \\
{ }_{D}\left\langle 0\left|\left[J_{V}^{1}(x), E(z)\right]\right| 0\right\rangle_{D}=\frac{i e^{2} \hbar}{\pi} \delta(x-z) .
\end{gathered}
$$




\section{THE ZERO MODE}

The simplest state with energy above the Dirac sea is the one associated to the only degree of freedom of the electromagnetic field which cannot be gauged away: the zero mode. Such a state can be neatly described in the present formalism as follows. Recalling that $c=\frac{1}{L} \int_{0}^{L} d z A(z)$, and $-\frac{\pi}{e L} \leq c \leq \frac{\pi}{e L}$ it is useful to consider the transformation

$$
\left|c, x_{1}, y_{1}, \ldots, x_{a}, y_{a}\right\rangle=\sum_{n} \mathrm{e}^{-i e c\left[n L+\sum_{k}\left(y_{k}-x_{k}\right)\right]}\left|n, x_{1}, y_{1}, \ldots, x_{a}, y_{a}\right\rangle
$$

Accordingly, the action of the relevant operators becomes

$$
\begin{aligned}
T^{0}[\gamma]\left|c, x_{1}, y_{1}, \ldots, x_{a}, y_{a}\right\rangle & =\mathrm{e}^{i e n_{\gamma} c L}\left|c, x_{1}, y_{1}, \ldots, x_{a}, y_{a}\right\rangle \\
\Pi_{0}(x, y)\left|c, x_{1}, y_{1}, \ldots, x_{a}, y_{a}\right\rangle & =\mathrm{e}^{i e c(y-x)}\left|c, x, y, x_{1}, y_{1}, \ldots, x_{a}, y_{a}\right\rangle \\
\Pi_{1}(x, y)\left|c, x_{1}, y_{1}, \ldots, x_{a}, y_{a}\right\rangle & =\mathrm{e}^{i e c(y-x)} \sum_{i} \delta\left(y-x_{i}\right)\left|c, x_{1}, y_{1}, \ldots, x, y_{i}, \ldots, x_{a}, y_{a}\right\rangle \\
\Pi_{2}(x, y)\left|c, x_{1}, y_{1}, \ldots, x_{a}, y_{a}\right\rangle & =-\mathrm{e}^{i e c(y-x)} \sum_{i} \delta\left(x-y_{i}\right)\left|c, x_{1}, y_{1}, \ldots, x_{i}, y, \ldots, x_{a}, y_{a}\right\rangle \\
E(z)\left|c, x_{1}, y_{1}, \ldots, x_{a}, y_{a}\right\rangle & =\frac{i \hbar}{L} \frac{\partial}{\partial c}\left|c, x_{1}, y_{1}, \ldots, x_{a}, y_{a}\right\rangle \\
& +e \hbar\left[\sum_{k} \theta\left(x_{k}, y_{k}, z\right)-\sum_{k} \frac{\left(y_{k}-x_{k}\right)}{L}\right]\left|c, x_{1}, y_{1}, \ldots, x_{a}, y_{a}\right\rangle
\end{aligned}
$$

Translation of the boundary conditions in this representation yields

$$
\begin{aligned}
\left|c, \ldots, x_{i}, y_{i}+p L \ldots\right\rangle & =\mathrm{e}^{i p \pi} \sum_{n} \mathrm{e}^{i e c\left[(n+p) L+\sum_{k}\left(y_{k}-x_{k}\right)\right]}\left|n+p, \ldots, x_{k}, y_{k} \ldots\right\rangle \\
& =\mathrm{e}^{i p \pi}\left|c, \ldots, x_{i}, y_{i} \ldots\right\rangle
\end{aligned}
$$

The Hamiltonian can be rewritten now as

$$
\begin{aligned}
& H \Psi\left(c, x_{1}, y_{1}, \ldots, x_{a}, y_{a}\right)= \\
& \quad \frac{e^{2} \hbar^{2}}{2}\left\{\int_{0}^{L} d x\left[\frac{i}{e L} \frac{\partial}{\partial c}+\sum_{k}\left(\theta\left(x_{k}, y_{k} ; x\right)-\frac{\left(y_{k}-x_{k}\right)}{L}\right)\right]^{2}\right\} \Psi\left(c, x_{1}, y_{1}, \ldots, x_{a}, y_{a}\right) \\
& -i \hbar \lim _{\epsilon \rightarrow 0} \frac{\partial}{\partial \epsilon} \sum_{i}\left\{\mathrm{e}^{i e c \epsilon}\left[\Psi\left(c, \ldots, x_{i}, y_{i}-\epsilon, \ldots\right)+\Psi\left(c, \ldots, x_{i}+\epsilon, y_{i}, \ldots\right)\right]\right\} .
\end{aligned}
$$

Now it is convenient to disentangle the term

$$
X=\int_{0}^{L} d x\left[\frac{i}{e L} \frac{\partial}{\partial c}+\sum_{k}\left(\theta\left(x_{k}, y_{k} ; x\right)-\frac{\left(y_{k}-x_{k}\right)}{L}\right)\right]^{2} \equiv X_{1}+X_{2},
$$

in Eq. (137), where we recall that

$$
\bar{\theta}\left(x_{k}, y_{k} ; x\right) \equiv \theta\left(x_{k}, y_{k} ; x\right)-\frac{y_{k}-x_{k}}{L}=\frac{1}{L} \sum_{p \neq 0} \frac{1}{i p}\left(e^{i p y_{k}}-e^{i p x_{k}}\right) e^{-i p x},
$$

according to Eq.(20). Since the summation in the above equation is over $p \neq 0$, the integration of the crossed term in the square of Eq. (138) is zero. The integration of the first square term is immediate, leading to 


$$
X_{1}=-\frac{1}{e^{2} L} \frac{\partial^{2}}{\partial c^{2}}
$$

The integration of the second square term reduces to

$$
\begin{aligned}
X_{2} & =\int_{0}^{L} d x \sum_{k, l} \bar{\theta}\left(x_{k}, y_{k} ; x\right) \bar{\theta}\left(x_{l}, y_{l} ; x\right) \\
& =L \sum_{k, l}\left(V\left(y_{k}-y_{l}\right)+V\left(x_{k}-x_{l}\right)-V\left(y_{k}-x_{l}\right)-V\left(x_{k}-y_{l}\right)\right),
\end{aligned}
$$

where

$$
V(z)=\sum_{n \neq 0} \frac{1}{4 \pi^{2} n^{2}} e^{\frac{2 \pi i n}{L} z}
$$

The main conclusion of the above calculation is the fact the the solutions of the whole problem satisfy the separability condition

$$
\Psi\left(c, \ldots x_{I}, y_{I}, \ldots\right)=\Phi(c) \Theta\left(\ldots, x_{I}, y_{I}, \ldots\right) .
$$

The external field analysis performed in Section IV corresponds to neglecting the quadratic (electric field) term in (137) with respect to the fermionic contribution involving the $\epsilon \rightarrow 0$ limit. The lowest energy state should be associated to the Dirac sea and the corresponding energy is $E(c)=-\frac{\hbar \pi}{6 L}+\frac{\hbar L}{2 \pi} e^{2} c^{2},-\frac{\pi}{e L} \leq c \leq \frac{\pi}{e L}$.

The next step is to consider the zero-mode sector of the theory, which consists in taking $E(z) \rightarrow E_{0}=\frac{1}{L} \int d z E(z)$ in the Hamiltonian (137). It is important to remark that the separability of Eq. (137) in the form $\Psi\left(c, \ldots x_{I}, y_{I} \ldots\right)=\Phi(c) \Theta\left(\ldots x_{I}, y_{I} \ldots\right)$, implies that, in the compact case, the zero mode spectrum is a part of the exact spectrum of the full Schwinger model, in complete analogy with the noncompact case.

From the general expression (135), and recalling Eq. (20) we readily verify that

$$
E_{0}=\frac{i \hbar}{L} \frac{\partial}{\partial c}
$$

in this representation. It is a general property that $E_{0}|0\rangle_{D}=0$, which means that $\Theta_{D}=$ $\left\langle c, \ldots, x_{I}, y_{I} \ldots \mid 0\right\rangle_{D}$ is independent of the coordinate $c$.

Now, the contribution of the zero mode to the exact solution of the problem, corresponds to the choice

$$
\Psi\left(c, \ldots x_{I}, y_{I}, \ldots\right)=\Phi(c) \Theta_{D}\left(\ldots, x_{I}, y_{I}, \ldots\right) .
$$

Recalling that the fermionic part of the Hamiltonian acts like a derivative, the zero mode contribution $\Phi(c)$ fulfills the equation

$$
\left[-\frac{\hbar^{2}}{2 L} \frac{\partial^{2}}{\partial c^{2}}+\frac{\hbar L}{2 \pi} e^{2} c^{2}\right] \Phi(c)=E_{1} \Phi(c)
$$

where the constant $-\frac{\hbar \pi}{6 L}$ coming from the Dirac sea energy $E(c)$ was reabsorbed in $E_{1}$.

The inner product and the boundary conditions for the zero mode contributions are:

$$
\begin{aligned}
\langle\phi \mid \psi\rangle & =\int_{-\frac{\pi}{e L}}^{\frac{\pi}{e L}} d c \phi^{*}(c) \psi(c), \\
\phi\left(-\frac{\pi}{e L}\right) & =\phi\left(\frac{\pi}{e L}\right), \quad \phi^{\prime}\left(-\frac{\pi}{e L}\right)=\phi^{\prime}\left(\frac{\pi}{e L}\right), \quad-\frac{\pi}{e L} \leq c \leq \frac{\pi}{e L},
\end{aligned}
$$


in such a way that the electric field and the Hamiltonian are hermitian. Here the prime means the derivative of the corresponding function with respect to the argument.

The solution to the eigenvalue equation (146) can be expressed in terms of cylindrical parabolic functions [23] upon the change of variables

$$
\begin{aligned}
& x:=\sqrt{\frac{2 e L}{\sqrt{\pi \hbar}}} c, \quad E_{1}=-\sqrt{\frac{\hbar^{3}}{\pi}} e a, \\
& \psi(c) \rightarrow y(x), \quad-x_{M} \leq x \leq x_{M}, \quad x_{M} \equiv \sqrt{\frac{2}{e L} \sqrt{\frac{\pi^{3}}{\hbar}}},
\end{aligned}
$$

where $x$ and $a$ are dimensionless quantities. This yields the equation

$$
y^{\prime \prime}-\left(\frac{1}{4} x^{2}+a\right) y=0
$$

The periodic boundary conditions on $y(x)$ are

$$
y\left(x_{M}\right)=y\left(-x_{M}\right), \quad y^{\prime}\left(x_{M}\right)=y^{\prime}\left(-x_{M}\right) .
$$

The general solution of Eq.(149) is

$$
y(x)=A \mathrm{e}^{-\frac{x^{2}}{4}} \mathrm{M}\left(\frac{a}{2}+\frac{1}{4}, \frac{1}{2}, \frac{x^{2}}{2}\right)+B x \mathrm{e}^{-\frac{x^{2}}{4}} \mathrm{M}\left(\frac{a}{2}+\frac{3}{4}, \frac{3}{2}, \frac{x^{2}}{2}\right),
$$

where $\mathrm{M}(A, B, z)$ is the confluent hypergeometric function. It will be convenient to introduce the new label

$$
\tilde{l}:=\frac{2 \pi^{3 / 2}}{x_{M}^{2}}=e L \hbar^{1 / 2}:=l \pi^{3 / 2}
$$

Now we separately discuss the even and odd solutions:

(i) Even solutions: in this case the periodic boundary conditions (150) are automatic on $y$ and imply

$$
y^{\prime}\left(x_{M}\right)=y^{\prime}\left(-x_{M}\right)=0 .
$$

This eigenvalue condition for Eq. (149) will determine the energy $E_{1}(a)$ as a function of $l$. The above condition can be written as

$$
\mathrm{M}\left(\frac{a}{2}+\frac{1}{4}, \frac{1}{2}, \frac{x_{M}^{2}}{2}\right)=(2 a+1) \mathrm{M}\left(\frac{a}{2}+\frac{5}{4}, \frac{3}{2}, \frac{x_{M}{ }^{2}}{2}\right)
$$

and it defines the function $a=a(l)$. This function can only be determined numerically for arbitrary $l$ and it is shown in Fig. 1.

The novel properties are:

1. $\lim _{L \rightarrow 0} a(l)=a(0)=-\frac{1}{2}-2 n, \quad n=0,1,2,3 \ldots$ which reproduces the even subset of the standard $\mathrm{U}(1)$ non compact case (i.e. $-\infty \leq c \leq \infty$ ). We will label the even functions $a(l)$ by the integer $2 n$ : $a_{2 n}(l)$. In Fig. 1 . we use the notation $E n$, i.e. $E 0, E 1, E 2, \ldots$, for the corresponding solutions. 
2. From the numerical calculation we find that $a_{0}$ is monotonously increasing and also that $\lim _{L \rightarrow \infty} a_{0}(l)=a_{0}(\infty)=0$. This last property is consistent with the fact that if $a$ remains finite when $l \rightarrow \infty$, then $a=0$.

3. The behavior of $a_{2 n}(l)$ for $l \rightarrow 0$ is

$$
a_{2 n}(l)=-\frac{1}{2}-2 n+\frac{2 \mathrm{e}^{-\frac{1}{l}}}{l^{\left(2 n+\frac{1}{2}\right)} n ! \Gamma\left(n+\frac{1}{2}\right)} .
$$

From the above equation we conclude that $\left.a_{2 n}^{\prime}(l)\right|_{l=0}=0$ and also that $a_{2 n}(l)$ is an increasing function near $l=0$.

4. The behavior for negative $a_{2 n}$, with large absolute value $\left(\left|a_{2 n}\right|>>1\right)$, is given by

$$
a_{2 n}(l)=-\frac{\pi^{2}}{2} n^{2} l, \quad n=1,2,3 \ldots,
$$

which can be readily observed in Fig.1.

(ii) Odd solutions: in this case the periodic boundary conditions (150) are automatic on $y^{\prime}$ and imply

$$
y\left(x_{M}\right)=y\left(-x_{M}\right)=0 .
$$

This eigenvalue condition is

$$
\mathrm{M}\left(\frac{a}{2}+\frac{3}{4}, \frac{3}{2}, \frac{x_{M}^{2}}{2}\right)=0
$$

and it defines the odd sector of the function $a=a(l)$. Again, this function can only be determined numerically for arbitrary $l$ and it is shown in Fig. 1. The properties are:

1. $\lim _{L \rightarrow 0} a(l)=a(0)=-\frac{1}{2}-(2 n-1), \quad n=1,2,3 \ldots$, which reproduces the odd subset of the standard $\mathrm{U}(1)$ non compact case (i.e. $-\infty \leq c \leq \infty)$. We will label the functions $a(l)$ by the integer $2 n-1: a_{2 n-1}(l)$. In Fig.1. we denote by $O n$, i.e. $O 1, O 2, \ldots$, the corresponding solutions.

2. From the numerical calculation we find that $a_{2 n-1}(l)$ are monotonously decreasing functions.

3. The behavior of $a_{2 n-1}(l)$ for $l \rightarrow 0$ is

$$
a_{2 n-1}(l)=-\frac{1}{2}-(2 n-1)-\frac{2 \mathrm{e}^{-\frac{1}{l}}}{l^{\left(2 n-\frac{1}{2}\right)}(n-1) ! \Gamma\left(n+\frac{1}{2}\right)} .
$$

From the above equation we conclude that $\left.a_{2 n-1}^{\prime}(l)\right|_{l=0}=0$ and also that $a_{2 n-1}(l)$ is a decreasing function near $l=0$.

4. The behavior for negative $a_{2 n-1}$ with large absolute value $\left(\left|a_{2 n-1}\right|>>8\right)$ is given by

$$
a_{2 n-1}(l)=-\frac{\pi^{2}}{2} n^{2} l, \quad n=1,2,3 \ldots,
$$

which again can be readily observed in the figure.

Let us notice that the asymptotic behavior (large $l$ ) of $a_{2 n}$ and $a_{2 n-1}$ coincide for $n=$ $1,2, \ldots$

Finally, we notice that all the states in the compact case are invariant under large gauge transformations. In particular, that is the case for the vacuum, and consequently the $\theta$ dependence does not appear. 


\section{CONCLUDING REMARKS}

In this paper we have analysed the two dimensional compact electrodynamics using the loop representation. The loop variables introduced here adapt naturally to the study of the compact version of the Schwinger model in the continuum, which, up to our knowledge, has not been previously discussed in the literature. These variables provide gauge invariant and intrinsically regularized non-local fields to describe the model. In terms of them, the quantum commutator algebra is constructed in an unambiguous manner. In particular, the current-current and electric field-current commutators are directly recovered. The choice of our basic non-local variables is such that the algebra involved in the anomalous commutators is already realized in terms of the classical Poisson brackets . We also obtain full operator expressions for the divergences of the charged and axial currents, which still need to be analyzed in the Hilbert space of the problem. This work also reports the spectrum of the zero mode sector of the compact Schwinger model, which is an exact piece of the full spectrum, due to the separability of the Schroedinger equation. The zero mode energy turns out to be completely different from the standard equally-spaced oscillator spectrum of the non-compact case. Even though bosonization seems to be still present, the zero mode spectrum suggests that the compact system will not behave as a free massive scalar field.

The invariance of the theory under large gauge transformations insures the uniqueness of the vacuum. The axial anomaly is still present, however as it was noticed by Jackiw [24], the presence of an axial anomaly is not necessarily related with a non trivial topological structure. Further studies are required to determine that, either the chiral charge may be redefined in a gauge invariant way, or the conservation of the axial current cannot be restored in the compact case. Our main obstacle to proceed with the solution of the full compact model is to obtain a complete set of solutions of the Schroedinger equation arising from the Hamiltonian (137), which is of the many-body type, with a linear kinetic energy term and pairwise interactions given by the non-trivial potential (142). There remains also the task of giving a complete discussion of the structure of the Hilbert space. These problems are now under investigation.

Again, we emphasize that the choice between the compact or noncompact version of any gauge theory is just a mater of convenience, which should be ultimately decided in terms of the experimental consequences of each model. In this spirit, what is really missing in the vast literature regarding the Schwinger model is, up to our knowledge, a study of its compact version using one's favorite method of solution. The main difference between the compact and the noncompact version is expected to appear in the boundary conditions satisfied by the corresponding wave functions, as opposed to the form of the (functional) differential equations involved, which should be the same in both cases. This is in complete analogy with the case of a free particle in a line (noncompact case) compared with the free onedimensional rotator (compact case), both of which are governed by the same Schroedinger equation.

An alternative route in understanding the compact Schwinger model is been pursued in Ref. [25], following the method of Ref. [8]. Some preliminary results are the following. Since the Gauss law still implies that the wave function is independent of the excited modes of the vector potential in the Weyl gauge, there are no boundary condition modifications related to these variables arising from the compactification. The wave function depends only upon the zero mode of the vector potential and the full Hamiltonian is still separable into zero plus excited modes with the same Schroedinger equations as in the noncompact case. The zero mode wave function satisfies new boundary conditions, imposed by the compactification of the zero mode vector potential, leading to the same non-linearly spaced spectrum derived in the present work. This piece of the spectrum cannot be interpreted as a collection of particles with mass $\frac{e}{\sqrt{\pi}}$ at zero momentum. The study of the complete spectrum and the full Hilbert space of the compact Schwinger model, using this approach, is also in progress. 25 


\section{ACKNOWLEDGMENTS}

Partial support from CONACyT grant 3141-PE to HAMT, LFU and JDV is acknowledged. HAMT would also like to thank CLAF-México, the organizers of "Quantum Gravity in the Southern Cone" and ICTP-Trieste for hospitality and financial support at different stages of the present work. HAMT and LFU are also indebted to R. Gambini for the hospitality extended to them at the Departamento de Física de la Universidad de la República, Uruguay. LFU and JDV are supported by the grant DGAPA-IN100694 and DGAPA-IN100397. We thank Román Linares for verifying some of the calculations. RG acknowledges useful conversations with H. Fort regarding compact electrodynamics.

\section{APPENDIX A}

Using the scalar product given in Eq.(71), we are able to solve for the creationannihilation operators in terms of the fields $\psi_{1}, \psi_{2}$

$$
\begin{array}{ll}
a_{m}=e^{\frac{i}{\hbar} \epsilon_{m} t}\left(\phi_{m}, \psi_{1}\right), & a_{m}^{\dagger}=e^{-\frac{i}{\hbar} \epsilon_{m} t}\left(\phi_{m}^{*}, \psi_{1}^{*}\right), \\
b_{m}=e^{\frac{i}{\hbar} \epsilon_{m} t}\left(\phi_{m}^{*}, \psi_{2}^{*}\right), \quad b_{m}^{\dagger}=e^{-\frac{i}{\hbar} \epsilon_{m} t}\left(\phi_{m}, \psi_{2}\right) .
\end{array}
$$

In this way, all bilinear expressions containing creation-annihilation operators can be written in terms of the gauge-invariant operators $\Pi(x, y)$ defined at $t=0$.

A direct calculation shows that

$$
\begin{aligned}
& \Pi_{0}^{m n}=a_{m}^{\dagger} b_{n}^{\dagger}, \\
& \Pi_{1}^{m n}=a_{m}^{\dagger} a_{n}, \\
& \Pi_{2}^{m n}=-b_{n}^{\dagger} b_{m}, \\
& \prod_{3}^{m n}=b_{m} a_{n} .
\end{aligned}
$$

The fermionic character of the operators involved imply the following important symmetry properties of the above operators

$$
\Pi_{a}^{m n} \Pi_{a}^{m k}=0=\Pi_{a}^{m n} \Pi_{a}^{k m}, \quad a=0,3,
$$

which is reflected through $\Pi_{a}(x, y) \Pi_{a}(x, z)=0=\Pi_{a}(x, y) \Pi_{a}(z, y)$ in coordinate space. Another important related symmetry of the external field is $U(u, v) U(x, y)=U(u, y) U(x, v)$, which results because of the abelian character of the $U(1)$ connection. 


\section{REFERENCES}

[1] J. Schwinger, Phys. Rev. 125, 397 (1962); ibid 128, 2425 (1962).

2] L.S. Brown, Nuovo Cim. XXIX 3727 (1963); J.H. Lowenstein and J.A. Swieca, Ann. Phys. 68172 (1971); E. Abdalla and M.C.A. Abdalla, Non-perturbative methods in 2 dimensional quantum field theory (World Scientific, Singapore, 1991).

[3] For a recent review of the Schwinger model see for example C. Adams, Anomaly and Topological aspects of two-dimensional quantum electrodynamics, Dissertation, Universitat Wien, october 1993.

[4] N.S. Manton, Ann. Phys. 159, 220 (1985).

5. J.E. Hetrick and Y. Hosotani, Phys. Rev. D38, 2621 (1988).

6 M.A. Shifman, Phys. Rep. 209, 341 (1991).

7. R. Link, Phys. Rev. D42, 2103 (1990).

8 S. Iso and H. Murayama, Prog. Theo. Phys. 84,142 (1990).

9] J. Hallin and P. Liljenberg, Phys. Rev. D54, 1723 (1996); J. Hallin, QED Q $_{1+1}$ by Dirac Quantization, Preprint Göteborg, ITP 93-8, hep-th/9304101, May 1993.

[10] R. Gambini and J. Pullin, Loops, knots, gauge theories and quantum gravity (Cambridge: Gambridge Univ. Press 1996).

[11] C. Rovelli and L. Smolin, Phys. Rev. Lett. 61, 1155 (1988); Nucl. Phys. B133, 80 (1990).

[12] R. Gambini, Phys. Lett. B235 , 180 (1991).

13 R. Gambini and A. Trias, Phys. Rev. D 23, 553 (1981); Nucl. Phys. B256, 479 (1986).

14 H. Fort and R. Gambini, Phys. Rev. D 44, 1257 (1991).

15] H.A. Morales-Técotl and C. Rovelli, Phys. Rev. Lett. 72, 3642 (1994); Nucl. Phys. B451, 325 (1995).

[16] A.M. Polyakov, Gauge Fields and Strings (New York: Harwood 1987).

17. F. Palumbo, Phys. Letts. B225(1989)407.; ibid. B335(1994)215.

18] J.M.Aroca, H.Fort and Gonzalo Alvarez, Finite lattice hamiltonian computations in the P-representation: the Schwinger model, preprint hep-lat/9711049.

[19] H.Fort, The worldsheet formulation as an alternative method for simulating dynamical fermions, preprint hep-lat/9710082.

[20] A.M. Polyakov, Nucl. Phys. B486, 23 (1997).

21] H.Fort and R. Gambini, The U(1) and strong CP problem from the loop formulation perspective, preprint hep-th/9711174.

[22] J. Schwinger, Phys. Rev. Letts. 3, 296 (1959).

23] M. Abramowitz and I. A. Stegun, Handbook of Mathematical Functions (New York: Dover 1965).

[24] R. Jackiw Topological investigations on quantized gauge theories. Relativité Groupes et Topologie II, Les Houches 1983 B. DeWitt and R. Stora, Editors (North-Holland, Amsterdam 1986)

[25] R. Linares, H. A. Morales, L. F. Urrutia and J. D. Vergara, work in progress. 


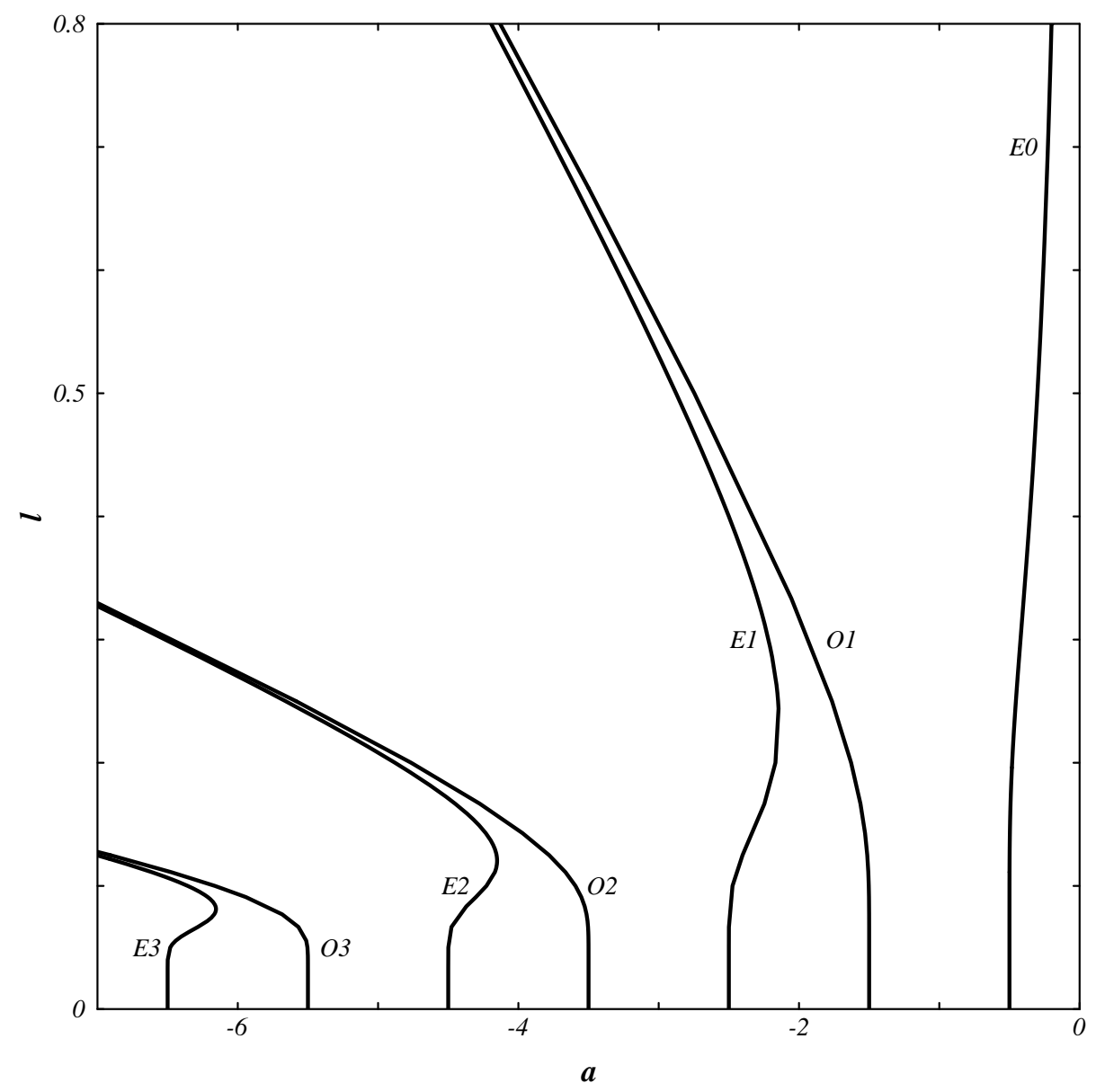

FIG.1. For a given value of $l$, the above curves provide the numerical solution for the parameter $a(l)$, which is related to the zero-mode spectrum of the system through $E_{1}=-\left(\hbar^{3} / \pi\right)^{1 / 2} e a(l)$. The even (odd) solutions are labeled by $E n(O n), n=0,1,2, \ldots$, and correspond to the solutions $a_{2 n}(l)\left(a_{2 n-1}(l)\right)$ of Section VII. 